\title{
Frames, operator representations, and open problems
}

\author{
Christensen, Ole; Hasannasab, Marzieh
}

Published in:

Operator Theory: Advances and Applications

Link to article, DOI:

10.1007/978-3-319-75996-8_8

Publication date:

2018

Document Version

Peer reviewed version

Link back to DTU Orbit

Citation (APA):

Christensen, O., \& Hasannasab, M. (2018). Frames, operator representations, and open problems. In Operator Theory: Advances and Applications (Vol. 268, pp. 155-165). Springer. Operator Theory Vol. 268 https://doi.org/10.1007/978-3-319-75996-8 8

\section{General rights}

Copyright and moral rights for the publications made accessible in the public portal are retained by the authors and/or other copyright owners and it is a condition of accessing publications that users recognise and abide by the legal requirements associated with these rights.

- Users may download and print one copy of any publication from the public portal for the purpose of private study or research.

- You may not further distribute the material or use it for any profit-making activity or commercial gain

- You may freely distribute the URL identifying the publication in the public portal

If you believe that this document breaches copyright please contact us providing details, and we will remove access to the work immediately and investigate your claim 


\title{
Frames, operator representations, and open problems
}

\author{
Ole Christensen and Marzieh Hasannasab
}

\begin{abstract}
A frame in a Hilbert space $\mathcal{H}$ is a countable collection of elements in $\mathcal{H}$ that allows each $f \in \mathcal{H}$ to be expanded as an (infinite) linear combination of the frame elements. Frames generalize the wellknown orthonormal bases, but provide much more flexibility and can often be constructed with properties that are not possible for orthonormal bases. We will present the basic facts in frame theory with focus on their operator theoretical characterizations and discuss open problems concerning representations of frames in terms of iterations of a fixed operator. These problems come up in the context of dynamical sampling, a topic that has recently attracted considerably interest within harmonic analysis. The goal of the paper is twofold, namely, that experts in operator theory will explore the potential of frames, and that frame theory will benefit from insight provided by the operator theory community.
\end{abstract}

Mathematics Subject Classification (2010). $42 \mathrm{C} 15$.

Keywords. Frames, dual frames, dynamical sampling, operator theory.

\section{Introduction and motivation}

A coherent state is a (typically overcomplete) system of vectors in a Hilbert space $\mathcal{H}$. In general it is given by the action of a class of linear operators on a single element in the underlying Hilbert space. In particular, it could be given by iterated action of a fixed operator on a single element, i.e., as $\left\{T^{n} \varphi\right\}_{n=0}^{\infty}$ for some $\varphi \in \mathcal{H}$ and a linear operator $T: \mathcal{H} \rightarrow \mathcal{H}$. Coherent states play an important role in mathematical physics [20, 22], operator theory, and modern applied harmonic analysis $[14,7]$. In particular, a Gabor system (see the definition below) is a coherent state.

Systems of vectors on the form $\left\{T^{n} \varphi\right\}_{n=0}^{\infty}$ also appear in the more recent context of dynamical sampling [1, 2, 3, 23]. Key questions in this context are whether $\left\{T^{n} \varphi\right\}_{n=0}^{\infty}$ can form a basis or a frame when the operator $T$ belongs to a certain class of operators, e.g., normal operators or self-adjoint operators. We will postpone the formal definition of a frame to Section 2 and just 
mention that a frame is a collection of vectors in $\mathcal{H}$ that allows each $f \in \mathcal{H}$ to be expanded as an (infinite) linear combination of the frame elements. Frames are getting increasingly popular in applied harmonic analysis and signal processing, mainly due to the fact that they are much more flexible and easier to construct with prescribed properties than the classical orthonormal bases.

A different approach was taken in the papers $[9,10,12]$ : here, the starting point is a frame and the question is when and how it has a representation of the form

$\left\{T^{n} \varphi\right\}_{n=0}^{\infty}$ for some $\varphi \in \mathcal{H}$ and a bounded linear operator $T: \mathcal{H} \rightarrow \mathcal{H}$. (1.1)

We will give a short survey of some of the most important results concerning frame representations of the form (1.1). As inspiration for the reader we will also state a number of open problems.

The above questions will also be analyzed with a different indexing, i.e., considering systems on the form $\left\{T^{n} \varphi\right\}_{n=-\infty}^{\infty}$ instead of $\left\{T^{n} \varphi\right\}_{n=0}^{\infty}$. The indexing in terms of $\mathbb{Z}$ is natural for several well-known classes of frames, and the theoretical conditions for a frame having such a representation with a bounded operator $T$ are similar to the ones for systems indexed by $\mathbb{N}_{0}$. The change in indexing gives an interesting twist on the problem. For example, a shift-invariant system (see Section 4 for the definition) always has a representation $\left\{T^{n} \varphi\right\}_{n=-\infty}^{\infty}$ with a bounded operator $T$, but it does not have a representation with the indexing in (1.1). This observation indeed leads to one of the open problems, stated for the so-called Gabor frames in $L^{2}(\mathbb{R})$.

The paper is organized as follows. In Section 2 we will provide a very short survey on frame theory with focus on operator theoretical characterizations. Section 3 discusses representations of frames via iterated systems of operators acting on a single element in the underlying Hilbert space. Finally, in Section 4 we consider concrete classes of frames, namely, shift-invariant frames and Gabor frames, and discuss a number of open problems related to operator representations.

\section{Frame theory}

Let $\mathcal{H}$ denote a separable Hilbert space. A sequence $\left\{f_{k}\right\}_{k=1}^{\infty}$ in $\mathcal{H}$ is a frame for $\mathcal{H}$ if there exist constants $A, B>0$ such that

$$
A\|f\|^{2} \leq \sum_{k=1}^{\infty}\left|\left\langle f, f_{k}\right\rangle\right|^{2} \leq B\|f\|^{2} \quad \forall f \in \mathcal{H} ;
$$

it is a frame sequence if the stated inequalities hold for all $f \in \overline{\operatorname{span}}\left\{f_{k}\right\}_{k=1}^{\infty}$. The sequence $\left\{f_{k}\right\}_{k=1}^{\infty}$ is a Bessel sequence if at least the upper frame condition holds. Also, $\left\{f_{k}\right\}_{k=1}^{\infty}$ is called a Riesz sequence if there exist constants $A, B>0$ such that

$$
A \sum\left|c_{k}\right|^{2} \leq\left\|\sum c_{k} f_{k}\right\|^{2} \leq B \sum\left|c_{k}\right|^{2}
$$


for all finite scalar sequences $\left\{c_{k}\right\}_{k=1}^{\infty}$. A Riesz basis is a Riesz sequence $\left\{f_{k}\right\}_{k=1}^{\infty}$ for which $\overline{\operatorname{span}}\left\{f_{k}\right\}_{k=1}^{\infty}=\mathcal{H}$.

It is well-known that the above concepts have operator theoretical characterizations, see, e.g., [7]:

Theorem 2.1. Consider a sequence $\left\{f_{k}\right\}_{k=1}^{\infty}$ in a separable Hilbert space $\mathcal{H}$. Then the following hold:

(i) $\left\{f_{k}\right\}_{k=1}^{\infty}$ is a Bessel sequence if and only if the mapping $U:\left\{c_{k}\right\}_{k=1}^{\infty} \mapsto$ $\sum_{k=1}^{\infty} c_{k} f_{k}$ is well-defined from $\ell^{2}(\mathbb{N})$ to $\mathcal{H}$, i.e., the infinite series is convergent for all $\left\{c_{k}\right\}_{k=1}^{\infty} \in \ell^{2}(\mathbb{N})$; in the affirmative case the operator $U$ is linear and bounded.

(ii) $\left\{f_{k}\right\}_{k=1}^{\infty}$ is a frame if and only if the mapping $\left\{c_{k}\right\}_{k=1}^{\infty} \mapsto \sum_{k=1}^{\infty} c_{k} f_{k}$ is well-defined from $\ell^{2}(\mathbb{N})$ to $\mathcal{H}$ and surjective.

(iii) $\left\{f_{k}\right\}_{k=1}^{\infty}$ is a Riesz basis if and only if the mapping $\left\{c_{k}\right\}_{k=1}^{\infty} \mapsto \sum_{k=1}^{\infty} c_{k} f_{k}$ is well-defined from $\ell^{2}(\mathbb{N})$ to $\mathcal{H}$ and bijective.

Theorem 2.1 tells us that if $\left\{f_{k}\right\}_{k=1}^{\infty}$ is a Bessel sequence, then the synthesis operator defined by

$$
U: \ell^{2}(\mathbb{N}) \rightarrow \mathcal{H}, U\left\{c_{k}\right\}_{k=1}^{\infty}:=\sum_{k=1}^{\infty} c_{k} f_{k}
$$

is well-defined and bounded. A central role will be played by the kernel of the operator $U$, i.e., the subset of $\ell^{2}(\mathbb{N})$ given by

$$
N_{U}=\left\{\left\{c_{k}\right\}_{k=1}^{\infty} \in \ell^{2}(\mathbb{N}) \mid \sum_{k=1}^{\infty} c_{k} f_{k}=0\right\} .
$$

We will now state one more characterization of frames, in terms of a condition that is very similar to the Riesz basis condition, except that it only takes place on sequences in the orthogonal complement of the kernel of the operator $U$.

Lemma 2.2. A sequence $\left\{f_{k}\right\}_{k=1}^{\infty}$ in $\mathcal{H}$ is a frame for $\mathcal{H}$ with bounds $A, B$ if and only if the following conditions are satisfied:

(i) $\left\{f_{k}\right\}_{k=1}^{\infty}$ is complete in $\mathcal{H}$.

(ii) The synthesis operator $U$ is well defined on $\ell^{2}(\mathbb{N})$ and

$$
A \sum_{k=1}^{\infty}\left|c_{k}\right|^{2} \leq\left\|U\left\{c_{k}\right\}_{k=1}^{\infty}\right\|^{2} \leq B \sum_{k=1}^{\infty}\left|c_{k}\right|^{2}, \forall\left\{c_{k}\right\}_{k=1}^{\infty} \in N_{U}^{\perp} .
$$

The excess of a frame is the number of elements that can be removed yet leaving a frame. It is well-known that the excess equals $\operatorname{dim}\left(N_{U}\right)$; see [4].

Given a Bessel sequence $\left\{f_{k}\right\}_{k=1}^{\infty}$, the frame operator is defined by

$$
S: \mathcal{H} \rightarrow \mathcal{H}, S f:=U U^{*} f=\sum_{k=1}^{\infty}\left\langle f, f_{k}\right\rangle f_{k} .
$$


For a frame $\left\{f_{k}\right\}_{k=1}^{\infty}$, the frame operator is bounded, bijective, and selfadjoint; these properties immediately lead to the important frame decomposition

$$
f=S S^{-1} f=\sum_{k=1}^{\infty}\left\langle f, S^{-1} f_{k}\right\rangle f_{k} \quad \forall f \in \mathcal{H} .
$$

The sequence $\left\{S^{-1} f_{k}\right\}_{k=1}^{\infty}$ is also a frame; it is called the canonical dual frame.

One of the most striking properties of frames is that they can be overcomplete; the intuitive interpretation of this is that a frame might consist of more elements than necessary to span the Hilbert space. In practice, this means that if $\left\{f_{k}\right\}_{k=1}^{\infty}$ is a frame but not a basis, then there exists $\left\{g_{k}\right\}_{k=1}^{\infty} \neq\left\{S^{-1} f_{k}\right\}_{k=1}^{\infty}$ such that

$$
f=\sum_{k=1}^{\infty}\left\langle f, g_{k}\right\rangle f_{k} \quad \forall f \in \mathcal{H} .
$$

Any frame $\left\{g_{k}\right\}_{k=1}^{\infty}$ satisfying (2.5) for a given frame $\left\{f_{k}\right\}_{k=1}^{\infty}$ is called a dual frame of $\left\{f_{k}\right\}_{k=1}^{\infty}$. Note that if $\left\{f_{k}\right\}_{k=1}^{\infty}$ and $\left\{g_{k}\right\}_{k=1}^{\infty}$ are Bessel sequences with synthesis operators $U, V$, respectively, then (2.5) means precisely that

$$
U V^{*}=I \text {. }
$$

We refer to [7] and [18] for more information about frames and Riesz bases.

\section{Operator representations of frames}

Formulated in purely mathematical terms, dynamical sampling in a Hilbert space $\mathcal{H}$ deals with frame properties of sequences in $\mathcal{H}$ of the form $\left\{T^{n} \varphi\right\}_{n=0}^{\infty}$, where $\varphi \in \mathcal{H}$ and $T: \mathcal{H} \rightarrow \mathcal{H}$ is a linear operator. The purpose of this section is to give an introduction to the topic that allows to discuss a number of important open problems as well.

Considering a class $\mathcal{A}$ of operators $T: \mathcal{H} \rightarrow \mathcal{H}$, typical questions in dynamical sampling are as follows:

- Can $\left\{T^{n} \varphi\right\}_{n=0}^{\infty}$ be a basis for $\mathcal{H}$ for some $T \in \mathcal{A}, \varphi \in \mathcal{H}$ ?

- Can $\left\{T^{n} \varphi\right\}_{n=0}^{\infty}$ be a frame for $\mathcal{H}$ for some $T \in \mathcal{A}, \varphi \in \mathcal{H}$ ?

Unfortunately, the theory of dynamical sampling is full of no-go results. Let us state some of them here.

Theorem 3.1. Consider a bounded operator $T: \mathcal{H} \rightarrow \mathcal{H}$, and let $\varphi \in \mathcal{H}$. Then the following hold:

(i) If $T$ is normal, then $\left\{T^{n} \varphi\right\}_{n=0}^{\infty}$ can not be a basis [2];

(ii) If $T$ is unitary, then $\left\{T^{n} \varphi\right\}_{n=0}^{\infty}$ can not be a frame [3];

(iii) If $T$ is compact, then $\left\{T^{n} \varphi\right\}_{n=0}^{\infty}$ can not be a frame [12]. 
The first construction of a frame of the form $\left\{T^{n} \varphi\right\}_{n=0}^{\infty}$ was obtained in [1] and further discussed in [2,3]. Since it deals with a class of diagonalizable operators it is natural to formulate it for the Hilbert space $\ell^{2}(\mathbb{N})$, where the operator $T$ can be considered as a matrix:

Theorem 3.2. Consider a diagonal matrix $T=\left[a_{j k}\right]_{j, k \in \mathbb{N}}$ given by $a_{k k}=$ $\lambda_{k}, a_{j k}=0, j \neq k$, and a sequence $\varphi=\{\varphi(k)\}_{k \in \mathbb{N}} \in \ell^{2}(\mathbb{N})$. Then the system $\left\{T^{n} \varphi\right\}_{n=0}^{\infty}$ is a frame for $\ell^{2}(\mathbb{N})$ if and only if

(i) $\left|\lambda_{k}\right|<1$ for all $k \in \mathbb{N}$;

(ii) $\left|\lambda_{k}\right| \rightarrow 1$ as $k \rightarrow \infty$;

(iii) The sequence $\left\{\lambda_{k}\right\}_{k=1}^{\infty}$ satisfies the Carleson condition, i.e.,

$$
\inf _{n} \prod_{n \neq k} \frac{\left|\lambda_{k}-\lambda_{n}\right|}{\left|1-\lambda_{k} \overline{\lambda_{n}}\right|}>0
$$

(iv) $\varphi(k)=m_{k} \sqrt{1-\left|\lambda_{k}\right|^{2}}$ for a scalar-sequence $\left\{m_{k}\right\}_{k=1}^{\infty}$ that is bounded below away from zero and above.

In the affirmative case $\left\{T^{n} \varphi\right\}_{n=0}^{\infty}$ is overcomplete, i.e., not a basis.

The fact that the Carleson condition comes in as the key condition in Theorem 3.2 indicates that the result is based on deep results concerning interpolation theory in spaces of analytic functions. Note that a self-contained proof (just based on a single result in [24] and standard frame theory) was given later in [13].

The construction in Theorem 3.2 can be extended to diagonalizable matrices, but very little is known in the literature about how and when one can construct frames for general matrices. Let us formulate this as an open problem:

Problem 1: Identify a class of non-diagonalizable matrices $T=\left[a_{j k}\right]_{j, k \in \mathbb{N}}$ for which

(i) $T$ is a bounded operator on $\ell^{2}(\mathbb{N})$;

(ii) $\left\{T^{n} \varphi\right\}_{n=0}^{\infty}$ is a frame for $\ell^{2}(\mathbb{N})$ for some $\varphi \in \ell^{2}(\mathbb{N})$.

The class of frames that are known to be representable on the form $\left\{T^{n} \varphi\right\}_{n=0}^{\infty}$ for a bounded operator $T$ is indeed limited: except for the construction in Theorem 3.2, such a representation has only been obtained for nonredundant frames, i.e., Riesz bases [9].

Note that the way the key questions in dynamical sampling are formulated in the introduction puts the operator in the central spot: we start with an operator and ask for frame properties of the associated iterated system. The opposite approach was taken in the papers $[9,10,12]$, where we considered a given frame $\left\{f_{k}\right\}_{k=1}^{\infty}$ and ask for the existence of a representation of the form

$$
\left\{f_{k}\right\}_{k=1}^{\infty}=\left\{T^{n} f_{1}\right\}_{n=0}^{\infty},
$$

where $T: \operatorname{span}\left\{f_{k}\right\}_{k=1}^{\infty} \rightarrow \mathcal{H}$ is a bounded linear operator. 
The following result collects a generalization of results from the papers $[9,10,12]$. Indeed, for reasons that will become clear in Section 4, we will generalize (3.2) and ask for representations of the form

$$
\left\{f_{k}\right\}_{k=1}^{\infty}=\left\{a_{n} T^{n} f_{1}\right\}_{n=0}^{\infty},
$$

for some scalars $a_{n} \neq 0$ with $\sup _{n}\left|\frac{a_{n}}{a_{n+1}}\right|<\infty$ and a bounded linear operator $T: \operatorname{span}\left\{f_{k}\right\}_{k=1}^{\infty} \rightarrow \mathcal{H}$. We will see in the subsequent example that the technical condition on the scalars $a_{n}$ is necessary. Define the weighted rightshift operator on $\ell^{2}\left(\mathbb{N}_{0}\right)$ by

$$
\mathcal{T}_{\omega}: \ell^{2}\left(\mathbb{N}_{0}\right) \rightarrow \ell^{2}\left(\mathbb{N}_{0}\right), \mathcal{T}_{\omega}\left(c_{0}, c_{1}, \cdots\right)=\left(0, \frac{a_{0}}{a_{1}} c_{0}, \frac{a_{1}}{a_{2}} c_{1}, \cdots\right)
$$

Theorem 3.3. Consider a frame $\left\{f_{k}\right\}_{k=1}^{\infty}$ for an infinite-dimensional Hilbert space $\mathcal{H}$, and a sequence of nonzero scalars $\left\{a_{n}\right\}_{n=0}^{\infty}$ such that $\sup _{n}\left|\frac{a_{n}}{a_{n+1}}\right|<$ $\infty$. Then the followings hold:

(i) $\left\{f_{k}\right\}_{k=1}^{\infty}$ is linearly independent if and only if there exists a linear operator $T: \operatorname{span}\left\{f_{k}\right\}_{k=1}^{\infty} \rightarrow \mathcal{H}$ such that (3.3) is satisfied.

(ii) Assume that $\left\{f_{k}\right\}_{k=1}^{\infty}$ is linearly independent. Then the operator $T$ in (3.3) is bounded if and only the kernel $N_{U}$ of the synthesis operator is invariant under weighted right-shifts given as in (3.4); in particular $T$ is bounded if $\left\{f_{k}\right\}_{k=1}^{\infty}$ is a Riesz basis.

(iii) Assume that $\left\{f_{k}\right\}_{k=1}^{\infty}$ is linearly independent and overcomplete. If the operator $T$ in (3.3) is bounded, then $\left\{f_{k}\right\}_{k=1}^{\infty}$ has infinite excess.

Proof. The proof of $(i)$ is similar to the proof of the non-weighted case given in [9]. In order to prove $(i i)$, consider now the representation (3.3). Let us write the synthesis operator for the frame $\left\{a_{n} T^{n} f_{1}\right\}_{n=0}^{\infty}$ as

$$
U: \ell^{2}\left(\mathbb{N}_{0}\right) \rightarrow \mathcal{H}, U\left\{c_{n}\right\}_{n=0}^{\infty}=\sum_{n=0}^{\infty} c_{n} a_{n} T^{n} f_{1}
$$

Now, assume first that $T$ is bounded and $\left\{c_{n}\right\}_{n=0}^{\infty} \in N_{U}$. Then

$$
\begin{aligned}
U \mathcal{T}_{\omega}\left\{c_{n}\right\}_{n=0}^{\infty}=\sum_{n=1}^{\infty} \frac{a_{n-1}}{a_{n}} c_{n-1} a_{n} T^{n} f_{1} & =\sum_{n=0}^{\infty} c_{n} a_{n} T^{n+1} f_{1} \\
& =T \sum_{n=0}^{\infty} c_{n} a_{n} T^{n} f_{1}=0 .
\end{aligned}
$$

Therefore $\mathcal{T}_{\omega}\left\{c_{n}\right\}_{n=0}^{\infty} \in N_{U}$. Conversely, assume that $N_{U}$ is invariant under the weighted right-shift operator. We want to prove that $T$ is bounded. Consider an element $f \in \mathcal{H}$ that has a finite expansion in terms of the frame $\left\{a_{n} T^{n} f_{1}\right\}_{n=0}^{\infty}$, i.e., $f=\sum_{n=0}^{N} c_{n} a_{n} T^{n} f_{1}$ for some $N \in \mathbb{N}, c_{n} \in \mathbb{C}$. Letting $c_{n}=0$ for $n>N$, we consider $\left\{c_{n}\right\}_{n=0}^{\infty}$ as a sequence in $\ell^{2}\left(\mathbb{N}_{0}\right)$. Choose $\left\{d_{n}\right\}_{n=0}^{\infty} \in N_{U}$ and $\left\{r_{n}\right\}_{n=0}^{\infty} \in N_{U}^{\perp}$ such that $\left\{c_{n}\right\}_{n=0}^{\infty}=\left\{d_{n}\right\}_{n=0}^{\infty}+\left\{r_{n}\right\}_{n=0}^{\infty}$. 
Letting $A, B$ denote frame bounds for $\left\{f_{k}\right\}_{k=1}^{\infty}$, Lemma 2.2 implies that

$$
\begin{aligned}
\|T f\|^{2} & =\left\|T \sum_{n=0}^{N} c_{n} a_{n} T^{n} f_{1}\right\|^{2} \\
& =\left\|T \sum_{n=0}^{\infty} r_{n} a_{n} T^{n} f_{1}\right\|^{2}=\left\|\sum_{n=0}^{\infty} r_{n} a_{n} T^{n+1} f_{1}\right\|^{2} \\
& =\left\|\sum_{n=0}^{\infty} r_{n} \frac{a_{n}}{a_{n+1}} a_{n+1} T^{n+1} f_{1}\right\|^{2} \leq \underset{n}{\leq} \sup _{n}\left|\frac{a_{n}}{a_{n+1}}\right|^{2} \sum_{n=0}^{\infty}\left|r_{n}\right|^{2} \\
& \leq B A^{-1} \sup _{n}\left|\frac{a_{n}}{a_{n+1}}\right|^{2}\left\|\sum_{n=0}^{\infty} r_{n} a_{n} T^{n} f_{1}\right\|^{2}=B A^{-1} \sup _{n}\left|\frac{a_{n}}{a_{n+1}}\right|^{2}\|f\|^{2} .
\end{aligned}
$$

Thus $T$ is bounded, as claimed.

(iii) Note that if $\left\{f_{k}\right\}_{k=1}^{\infty}=\left\{a_{n} T^{n} f_{1}\right\}_{n=0}^{\infty}$, then for $k \geq 1$,

$$
f_{k+1}=a_{k} T^{k} f_{1}=\frac{a_{k}}{a_{k-1}} T\left(a_{k-1} T^{k-1} f_{1}\right)=\frac{a_{k}}{a_{k-1}} T f_{k} .
$$

Now, if $\left\{f_{k}\right\}_{k=1}^{\infty}$ has finite excess, then there exists some $K \in \mathbb{N} \cup\{0\}$ such that $\left\{f_{k}\right\}_{k=K}^{\infty}$ is an overcomplete frame sequence and $\left\{f_{k}\right\}_{k=K+1}^{\infty}$ is a Riesz sequence. Therefore there exists a non-zero sequence $\left\{c_{k}\right\}_{k=K}^{\infty}$ such that $\sum_{k=K}^{\infty} c_{k} f_{k}=0$. Since $T$ is bounded, we have

$$
0=\sum_{k=K}^{\infty} c_{k} T f_{k}=\sum_{k=K}^{\infty} c_{k} \frac{a_{k-1}}{a_{k}} f_{k+1}=\sum_{k=K+1}^{\infty} c_{k-1} \frac{a_{k-2}}{a_{k-1}} f_{k} .
$$

Therefore $c_{k}=0$ for $k \in\{K, K+1, \cdots\}$ which is a contradiction. So $T$ can not be bounded.

Example. Consider any Riesz basis $\left\{f_{k}\right\}_{k=1}^{\infty}$ for $\mathcal{H}$, with frame bounds $A, B>$ 0 . Theorem 3.3 implies that for any sequence of nonzero scalars $\left\{a_{n}\right\}_{n=0}^{\infty}$ such that

$$
\sup _{n}\left|\frac{a_{n}}{a_{n+1}}\right|<\infty
$$

there exists a bounded operator $T: \mathcal{H} \rightarrow \mathcal{H}$ such that $\left\{f_{k}\right\}_{k=1}^{\infty}=\left\{a_{n} T^{n} f_{1}\right\}_{n=0}^{\infty}$. The condition (3.6) is indeed necessary for this conclusion to hold. Too see this, note first that if $\left\{f_{k}\right\}_{k=1}^{\infty}=\left\{a_{n} T^{n} f_{1}\right\}_{n=0}^{\infty}$, then as we saw in (3.5) we have $T f_{k}=\frac{a_{k-1}}{a_{k}} f_{k+1}$. Using that $\sqrt{A} \leq\left\|f_{k}\right\| \leq \sqrt{B}$ for all $k \in \mathbb{N}$, it follows that

$$
\left\|T f_{k}\right\|=\left\|\frac{a_{k-1}}{a_{k}} f_{k+1}\right\| \geq\left|\frac{a_{k-1}}{a_{k}}\right| \sqrt{A} \geq\left|\frac{a_{k-1}}{a_{k}}\right| \sqrt{\frac{A}{B}}\left\|f_{k}\right\|,
$$

which implies that $T$ is unbounded if (3.6) is violated. 


\subsection{Cyclic vectors and hypercyclic vectors}

We would like to point out that the frame condition on an iterated system $\left\{T^{n} \varphi\right\}_{n=0}^{\infty}$ is indeed very different from the conditions that are typically considered in operator theory for such systems. First, consider a linear operator $T: \mathcal{H} \rightarrow \mathcal{H}$ and recall that a vector $\varphi \in \mathcal{H}$ is said to be cyclic with respect to $T$ if $\operatorname{span}\left\{T^{n} \varphi\right\}_{n=0}^{\infty}=\mathcal{H}$. By (2.4) this condition is satisfied if $\left\{T^{n} \varphi\right\}_{n=0}^{\infty}$ is a frame for $\mathcal{H}$. However, the frame condition is significantly stronger than the condition of $\varphi$ being cyclic. In order to illustrate this, let $\left\{e_{k}\right\}_{k=1}^{\infty}$ denote an orthonormal basis for $\mathcal{H}$ and consider the family $\left\{f_{k}\right\}_{k=1}^{\infty}:=\left\{e_{k}+e_{k+1}\right\}_{k=1}^{\infty}$. Define the operator $T$ by $T e_{k}:=e_{k+1}$; then $T$ can be extended to a bounded linear operator on $\mathcal{H}$ and $\left\{f_{k}\right\}_{k=1}^{\infty}=\left\{T^{n}\left(e_{1}+e_{2}\right)\right\}_{n=0}^{\infty}$. It is known (see Example 5.4.6 in [7]) that $\overline{\operatorname{span}}\left\{f_{k}\right\}_{k=1}^{\infty}=\mathcal{H}$, which implies that the vector $\varphi:=e_{1}+e_{2}$ is cyclic with respect to the operator $T$; however, the same example in [7] shows that $\left\{T^{n}\left(e_{1}+e_{2}\right)\right\}_{n=0}^{\infty}$ is not a frame.

Recall also that a vector $\varphi \in \mathcal{H}$ is hypercyclic with respect to the operator $T$ if $\left\{T^{n} \varphi\right\}_{n=0}^{\infty}$ is dense in $\mathcal{H}$. This condition is way too strong in the context of frames, as it implies that $\left\{T^{n} \varphi\right\}_{n=0}^{\infty}$ does not satisfy the Bessel condition.

\section{Shift-invariant systems and Gabor frames}

In this section we will consider some classes of explicitly given frames in the Hilbert space $L^{2}(\mathbb{R})$ and open problems related to dynamical sampling. For our purpose the central class of frames is the so-called Gabor frames, but it is natural also to consider shift-invariant systems. Both systems are defined in terms of certain classes of operators on $L^{2}(\mathbb{R})$.

For $a \in \mathbb{R}$, define the translation operator

$$
T_{a}: L^{2}(\mathbb{R}) \rightarrow L^{2}(\mathbb{R}), T_{a} f(x):=f(x-a)
$$

and the modulation operator

$$
E_{a}: L^{2}(\mathbb{R}) \rightarrow L^{2}(\mathbb{R}), E_{a} f(x):=e^{2 \pi i a x} f(x) .
$$

The translation operators and the modulation operators are unitary. We define the Fourier transform of $f \in L^{1}(\mathbb{R})$ by

$$
\widehat{f}(\gamma)=\mathcal{F} f(\gamma)=\int_{-\infty}^{\infty} f(x) e^{-2 \pi i \gamma x} d x
$$

and extend it in the standard way to a unitary operator on $L^{2}(\mathbb{R})$.

Given a function $\varphi \in L^{2}(\mathbb{R})$ and some $b>0$, the associated shiftinvariant system is given by $\left\{T_{k b} \varphi\right\}_{k \in \mathbb{Z}}$. The frame properties of such systems are well understood. The following proposition collects some of the main results. Given $\varphi \in L^{2}(\mathbb{R})$ and some $b>0$, consider the function

$$
\Phi(\gamma):=\sum_{k \in \mathbb{Z}}\left|\widehat{\varphi}\left(\frac{\gamma+k}{b}\right)\right|^{2}, \gamma \in \mathbb{R} .
$$


Proposition 4.1. Let $\varphi \in L^{2}(\mathbb{R}) \backslash\{0\}$ and $b>0$ be given. Then the following hold:

(i) $\left\{T_{k b} \varphi\right\}_{k \in \mathbb{Z}}$ is linearly independent.

(ii) $\left\{T_{k b} \varphi\right\}_{k \in \mathbb{Z}}$ is a Riesz basis if and only if there exist $A, B>0$ such that $A \leq \Phi(\gamma) \leq B$ for almost all $\gamma \in[0,1]$.

(iii) $\left\{T_{k b} \varphi\right\}_{k \in \mathbb{Z}}$ is a frame sequence if and only if there exist $A, B>0$ such that $A \leq \Phi(\gamma) \leq B$ for almost all $\gamma \in[0,1] \backslash\{\gamma \in[0,1] \mid \Phi(\gamma)=0\}$.

(iv) $\left\{T_{k b} \varphi\right\}_{k \in \mathbb{Z}}$ can at most be a frame for a proper subspace of $L^{2}(\mathbb{R})$.

(v) If $\left\{T_{k b} \varphi\right\}_{k \in \mathbb{Z}}$ is an overcomplete frame sequence, it has infinite excess.

(vi) $\left\{T_{k b} \varphi\right\}_{k \in \mathbb{Z}}=\left\{\left(T_{b}\right)^{k} \varphi\right\}_{k \in \mathbb{Z}}$, i.e., the system $\left\{T_{k b} \varphi\right\}_{k \in \mathbb{Z}}$ has the form of an iterated system indexed by $\mathbb{Z}$.

The result in (i) is well-known, and (ii) \& (iii) are proved in [5]; (iv) is proved in [8], (v) is proved in [4, 10], and (vi) is clear.

Note that the representation of a shift-invariant system as an iterated system in Proposition 4.1 (v) differs from the ones appearing in Section 3 in terms of the index set $\mathbb{Z}$. It was recently shown in [11] that even though a shift-invariant frame $\left\{T_{k b} \varphi\right\}_{k \in \mathbb{Z}}$ can be re-indexed and be represented in the form (1.1) for a linear operator $T: \operatorname{span}\left\{T_{k b} \varphi\right\}_{k \in \mathbb{Z}} \rightarrow L^{2}(\mathbb{R})$, the operator $T$ can only be bounded if $\left\{T_{k b} \varphi\right\}_{k \in \mathbb{Z}}$ is a basis.

Let us now introduce the Gabor systems. Given some $a, b>0$ and a function $g \in L^{2}(\mathbb{R})$, the associated Gabor system is the collection of functions given by

$$
\left\{E_{m b} T_{n a} g\right\}_{m, n \in \mathbb{Z}}=\left\{e^{2 \pi i m b x} g(x-n a)\right\}_{m, n \in \mathbb{Z}} .
$$

Gabor systems play an important role in time-frequency analysis; we will just state the properties that are necessary for the flow of the current paper, and refer to $[17,15,16,7]$ for much more information.

Proposition 4.2. Let $g \in L^{2}(\mathbb{R}) \backslash\{0\}$. Then the following hold:

(i) $\left\{E_{m b} T_{n a} g\right\}_{m, n \in \mathbb{Z}}$ is linearly independent.

(ii) If $\left\{E_{m b} T_{n a} g\right\}_{m, n \in \mathbb{Z}}$ is a frame for $L^{2}(\mathbb{R})$, then $a b \leq 1$.

(iii) If $\left\{E_{m b} T_{n a} g\right\}_{m, n \in \mathbb{Z}}$ is a frame for $L^{2}(\mathbb{R})$, then $\left\{E_{m b} T_{n a} g\right\}_{m, n \in \mathbb{Z}}$ is a Riesz basis if and only if $a b=1$.

(iv) If $\left\{E_{m b} T_{n a} g\right\}_{m, n \in \mathbb{Z}}$ is an overcomplete frame for $L^{2}(\mathbb{R})$, then it has infinite excess.

The result in (i) was proved in [21] (hereby confirming a conjecture stated in [19]); (ii) \& (iii) are classical results [17, 7], and (iv) is proved in [4].

Note that since a Gabor frame $\left\{E_{m b} T_{n a} g\right\}_{m, n \in \mathbb{Z}}$ is linearly independent by Proposition 4.2 (i), Proposition 3.3 shows that any reordering $\left\{f_{k}\right\}_{k=1}^{\infty}$ can be represented on the form $\left\{T^{n} \varphi\right\}_{n=0}^{\infty}$ for some linear operator $T: L^{2}(\mathbb{R}) \rightarrow$ $L^{2}(\mathbb{R})$ and some $\varphi \in L^{2}(\mathbb{R})$. However, it was recently shown in [11] that the operator $T$ always is unbounded, except in the case where the Gabor frame is a Riesz basis, i.e., if $a b=1$. This is indeed the reason that we considered the more general representations (3.3) in Section 3: the hope is that the 
possibility of choosing appropriate coefficients $a_{n}$ allows to find a bounded operator $T$. Let us formulate the key question as an open problem:

Problem 2: Do there exist overcomplete Gabor frames $\left\{E_{m b} T_{n a} g\right\}_{m, n \in \mathbb{Z}}$ such that an appropriate ordering $\left\{f_{k}\right\}_{k=1}^{\infty}$ of the frame elements has a representation

$$
\left\{f_{k}\right\}_{k=1}^{\infty}=\left\{a_{n} T^{n} \varphi\right\}_{n=0}^{\infty},
$$

for some scalars $a_{n} \neq 0$, a bounded operator $T: L^{2}(\mathbb{R}) \rightarrow L^{2}(\mathbb{R})$, and some $\varphi \in L^{2}(\mathbb{R})$ ?

Note that if the Gabor frame $\left\{E_{m b} T_{n a} g\right\}_{m, n \in \mathbb{Z}}$ is generated by a function $g$ for which $\|g\|=1$ and we assume that $a_{n}>0$ for all $n \in \mathbb{N}_{0}$, the representation (4.2) implies that $a_{n}=\left\|T^{n} \varphi\right\|^{-1}$, and thus the representation takes the form

$$
\left\{f_{k}\right\}_{k=1}^{\infty}=\left\{\frac{T^{n} \varphi}{\left\|T^{n} \varphi\right\|}\right\}_{n=0}^{\infty},
$$

For shift-invariant frames $\left\{T_{k b} \varphi\right\}_{k \in \mathbb{Z}}$, we just saw that they are indeed "born" having the structure of an iterated system, indexed by $\mathbb{Z}$. Thus, it is natural to ask whether such a representation is possible for Gabor frames as well. Let us formulate this as the final problem:

Problem 3: Do there exist overcomplete Gabor frames $\left\{E_{m b} T_{n a} g\right\}_{m, n \in \mathbb{Z}}$ such that an appropriate ordering $\left\{f_{k}\right\}_{k=-\infty}^{\infty}$ of the frame elements has a representation

$$
\left\{f_{k}\right\}_{k=-\infty}^{\infty}=\left\{T^{n} \varphi\right\}_{n=-\infty}^{\infty}
$$

for some bounded operator $T: L^{2}(\mathbb{R}) \rightarrow L^{2}(\mathbb{R})$ and some $\varphi \in L^{2}(\mathbb{R})$ ?

Acknowledgment: The authors would like to thank Professor Albrecht Böttcher for many useful comments to the manuscript.

\section{References}

[1] Aldroubi, A., Cabrelli, C., Molter, U., and Tang, S.: Dynamical sampling. Appl. Harm. Anal. Appl. 42 no. 3 (2017), 378-401.

[2] Aldroubi, A., Cabrelli, C., Çakmak, A. F., Molter, U., and Petrosyan, A.: Iterative actions of normal operators. J. Funct. Anal. 272 no. 3 (2017), 11211146.

[3] Aldroubi, A., and Petrosyan, A.: Dynamical sampling and systems from iterative actions of operators. In "Frames and Other Bases in Abstract and Function Spaces." Eds. Mhaskar, H., Pesenson, I. Zhou, D. X., Le Gia, Q. T., and Mayeli, A., Birkhäuser, Boston, 2017

[4] Balan, R., Casazza, P., Heil, C. and Landau, Z.: Deficits and excesses of frames. Adv. Comp. Math. 18 (2002), 93-116. 
[5] Benedetto, J. and Li, S.: The theory of multiresolution analysis frames and applications to filter banks. Appl. Comp. Harm. Anal. 5 (1998), 389-427.

[6] Cabrelli, C., Molter, U, Paternostro, V., and Philipp, F.: Dynamical sampling on finite index sets. Preprint, 2017.

[7] Christensen, O.: An introduction to frames and Riesz bases. Second expanded edition. Birkhäuser, Boston, 2016.

[8] Christensen, O., Deng, B., and Heil, C.: Density of Gabor frames. Appl. Comp. Harm. Anal. 7 (1999), 292-304.

[9] Christensen, O., and Hasannasab, M.: Frame properties of systems arising via iterative actions of operators. Preprint, 2016.

[10] Christensen, O., and Hasannasab, M.: Operator representations of frames: boundedness, duality, and stability Integral Equations an Operator Theory $\mathbf{. 8 8}$ no. 4 (2017), 483-499.

[11] Christensen, O., Hasannasab, M., and Philipp, F.: Frame properties of operator orbits. Manuscript in progress.

[12] Christensen, O., Hasannasab, M., and Rashidi, E.: Dynamical sampling and frame representations with bounded operators. Preprint, 2017.

[13] Christensen, O., Hasannasab, M., and Stoeva, D. T.: Operator representations of sequences and dynamical sampling. Preprint, 2017.

[14] Daubechies, I.: The wavelet transformation, time-frequency localization and signal analysis. IEEE Trans. Inform. Theory 36 (1990), 961-1005.

[15] Feichtinger, H. G. and Strohmer, T. (eds.): Gabor Analysis and Algorithms: Theory and Applications. Birkhäuser, Boston, 1998.

[16] Feichtinger, H. G. and Strohmer, T. (eds.): Advances in Gabor Analysis. Birkhäuser, Boston, 2002.

[17] Gröchenig, K.: Foundations of time-frequency analysis. Birkhäuser, Boston, 2000 .

[18] Heil, C.: A basis theory primer. Expanded edition. Applied and Numerical Harmonic Analysis. Birkhäuser/Springer, New York, 2011.

[19] Heil, C., Ramanathan, J. and Topiwala, P.: Linear independence of timefrequency translates. Proc. Amer. Math. Soc. 124 (1996), 2787-2795.

[20] Klauder, J. and Skagerstam, B.: Coherent states. Applications in physics and mathematical physics. World Scientific, Singapore, 1985.

[21] Linnell, P.: Von Neumann algebras and linear independence of translates. Proc. Amer. Math. Soc. 127 no. 11 (1999), 3269-3277.

[22] Perelomov, A.: Generalized coherent states and their applications. Springer, New York, 2012.

[23] Philipp, F.: Bessel sequences from iterated operator actions. Preprint, 2016.

[24] Shapiro, H. S., and Shields, A. L.: On some interpolation problems for analytic functions. American Journal of Mathematics, 83 no.3 (1961), 513-532. 
Ole Christensen

Technical University of Denmark DTU Compute

Building 303, 2800 Lyngby

e-mail: ochr@dtu.dk

Marzieh Hasannasab

Technical University of Denmark DTU Compute

Building 303, 2800 Lyngby

e-mail: mhas@dtu.dk 